\title{
Precise Generators Synchronization a Small HPP with an Excitation System
}

\author{
Angel Tsolov \\ Faculty of Electrical Engineering \\ Technical University of Sofia \\ Sofia, Bulgaria \\ abc@tu-sofia.bg
}

\begin{abstract}
The main problem in the process of synchronization of small synchronous generators in HPPs is the maintenance of a constant difference in the angular velocities of the generator and the system (sliding). This is necessary for the correct operation of any automatic accurate synchronization algorithm. This article proposes a method based on the combined action of the excitation system and the speed regulator of synchronous generators. The results provide a basis for the practical implementation of the proposed algorithm relevant for mathematical and physical research.
\end{abstract}

Keywords-excitation system; constatnt leakage; synchronous generator; small HPP; multi channel regulator; mathematical model; physicall modeling; FEM; Simulink

\section{INTRODUCTION}

In classical methods and approaches, the possibility of accurate synchronization of a generator by using the mutual influence between the frequency controller and the excitation regulator is not considered. The idea is to use this for accurate synchronization, but the following conditions must be met: the generator and grid voltage must be approximately equal per module, the frequency of the generator and the grid must be different with necessary leakage between them, at the moment of switching on, the angle between the voltage of the generator and that of the grid must be minimal and the rotational direction of their fields must be the same $[1,2]$.

\section{PHYSICAL ESSENCE OF THE PROCESS}

\section{A. Antagonism Between Control Systems}

Two controllers work in the twist and synchronization process:

- The excitation system that adjusts the idle voltage.

- A speed governor that maintains the frequency leakage.

With steady speed adjustment, rising speed creates a slight increase in voltage. On the other side, this will cause the excitation regulator to react to adjust (reduce) the voltage. Figure 1 shows a record of a real process (HPP Garvanitca) that illustrates the situation. In the process of synchronization, one system has a disturbing effect on the other system and the synchronous generator $(\mathrm{SG})$ fails to come in parallel with the power system for the set time. In the event of a slight revolution increase by the action of the speed controller, the output voltage of the generator is also increased. In the same time the generator voltage regulator seeks to compensate for this variation by reducing the generator's excitation current and the flux respectively. As a secondary result, the revolutions increase further and this results in a new action in the opposite direction of the speed regulator.

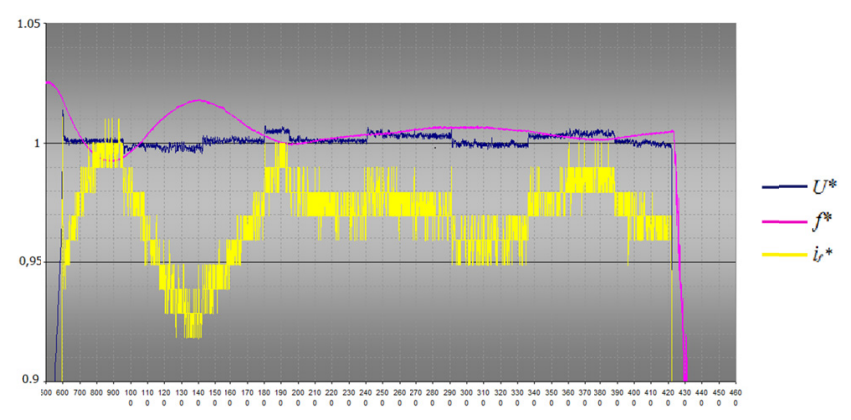

Fig. 1. Recording voltage, frequency and excitation current of synchronized generator at 1.3MVA, during synchronization process (HPP Garvanica, 2010)

Due to the large difference between the units of the mechanical $\left(\mathrm{T}_{J}\right)$ and the electrical $\left(\mathrm{T}_{d 0} \equiv \mathrm{T}_{e}\right.$ for hydro generators) time constants, a fluctuating process is reached which slows down gradually. In addition, disturbing impacts are occurring (different initial water pressure conditions, working of another machine on the common busbar), which generally hinder the synchronization.

\section{B. Processes Before Breaker On}

The synchronization process is shown schematically in Figure 2. The power system is replaced with an endless voltage bus $V_{p s}$ behind an equivalent reactive bulb $X_{p s}$. Immediately before closing the circuit breaker, it is correct to rotate at a velocity $\omega_{g}$ slightly bigger than the synchronous velocity $\omega_{p s}$ (with necessary sliding) and the excitation to provide an unloaded output voltage $E_{f}$ close to the system voltage $V_{p s}$. 


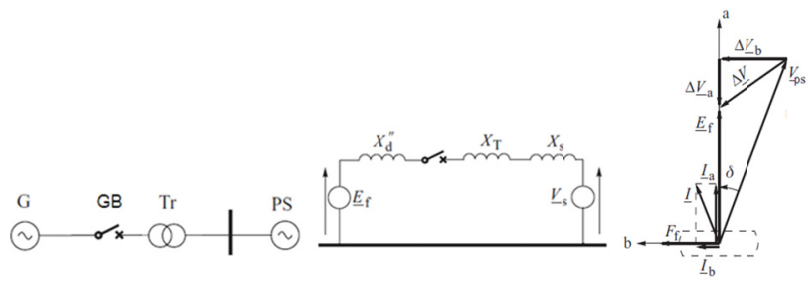

Fig. 2. Scheme of the synchronization process and phazor diagrame

The vector difference $d U$ depends on the absolute values of $E_{f}, \mathrm{~V}_{\mathrm{ps}}$, and the offset angle between them at the moment of switching on the GB switch (Figure 2) and is generally determined by the expression:

$$
d U=\sqrt{V_{p s}^{2}+E_{f}^{2}-2 E_{f} V_{p s} \cos \delta}
$$

From (1), in case of dephasation $\delta=\pi r a d\left(180^{\circ}\right)$ and the vectors $\mathrm{E}_{\mathrm{f}}$ and $V_{p s}$ are in the opposite direction, the vector difference $d U$ is maximal $\left(d U=d U_{\max }\right)$, and $\delta=0$. When they are in the same direction the difference is minimal $(d U=$ $\left.d U_{\min }\right)$ [3]. Provided the tensions are aligned in amplitude: $\left|\mathrm{E}_{\mathrm{f}}\right|=\left|\mathrm{V}_{\mathrm{ps}}\right|=|\mathrm{U}|$, then $d U_{\text {max }}=2 U \rightarrow(\delta=\pi)$ or $d U_{\text {min }}=0 \rightarrow(\delta=0)$.

In order to guarantee the flow of minimum equalization current, frequency details $f_{g}\left(\omega_{g}\right)$ and $f_{p s}\left(\omega_{p s}\right)$ and the angle between the vectors $\overrightarrow{E_{f}}$ and $\overrightarrow{V_{p s}}$ are required. Under the condition $\left|\mathrm{E}_{\mathrm{f}}\right|=\left|V_{p s}\right|=\left|\mathrm{U}_{\mathrm{m}}\right|, \mathrm{d} U$ turns into:

$$
\begin{aligned}
& d U=U_{m}\left(\sin \omega_{g} t-\sin \omega_{p s} t\right), \\
& d U=2 U_{m} \sin \left(\frac{\omega_{g}-\omega_{p s}}{2} t\right) \cos \left(\frac{\omega_{g}+\omega_{p s}}{2} t\right) .
\end{aligned}
$$

The voltage between the poles of the circuit breaker is sinusoidal with angular frequency $\omega_{s}=\frac{\omega_{g}+\omega_{p s}}{2}$, frequency $f_{s}=\omega / 2 \pi$ and beating period $T_{s}=1 / f_{s}$. The shell of this curve is called beating curve, which in case $\left|E_{f}\right|=\left|V_{p s}\right|$ and $\omega_{s} \neq 0$ is shown in Figure 3(a), and for $\left|E_{f}\right| \neq\left|V_{p s}\right|$ and $\omega_{s} \neq 0$ in Figure 3(b), at three different sliding frequencies $f_{s 1}>f_{s 2}>$ $f_{s 3} . \tau_{Y A C}$ indicates the own switching time of the GB switch. As $\tau_{\mathrm{YAC}}=$ const, command is given to turn on GB at a time when $\delta=\delta_{\text {nom }}$, the switching will be performed at dfferent $d U\left(d U_{1}, d U_{2}, d U_{3}\right)$, depending on $f_{s}$, (larger for bigger frequency differences (bigger leakage)).

\section{Processes After Breaker On}

Depending on the moment the GB breaker is switched on, an equalizing current will occur,

$$
I_{e}=\frac{E_{f}-V_{p s}}{j\left(x_{d}^{\prime \prime}-X_{p s}\right)}=\frac{d U}{j X_{\Sigma}}
$$

For dynamic efforts, the overcurrent $i_{e}$ is most defining:

$$
i_{e}^{\prime \prime}=\frac{d U * 1.8 \sqrt{2}}{j X_{\Sigma}}
$$

This depends on two main elements with sequential action:

- $\quad$ anchoring current of armature;

- current forced by the stored kinetic energy of the rotor.

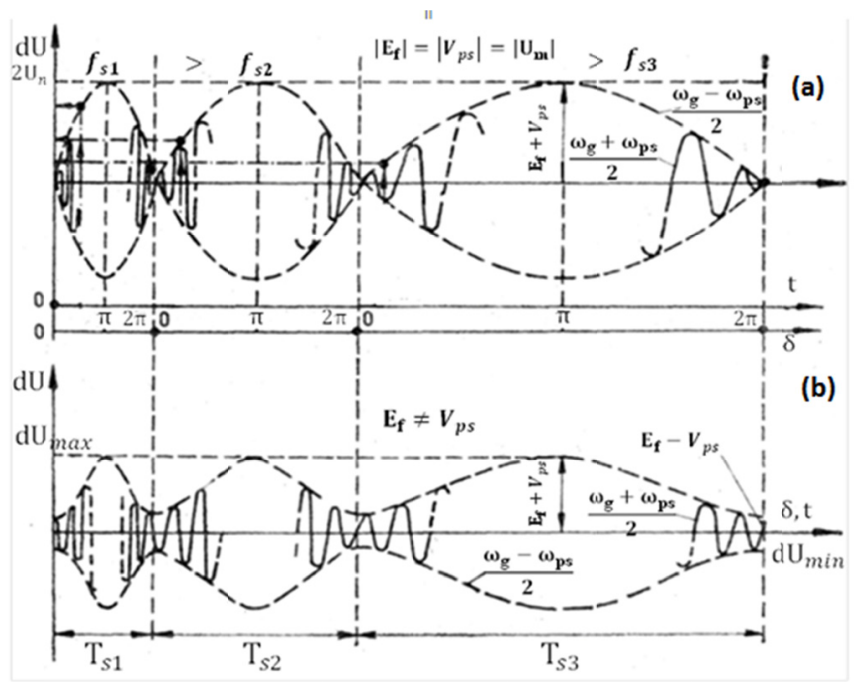

Fig. 3. The synchronization process - diagrame of the informative parameter dU for (a) $\left|E_{f}\right|=\left|V_{p s}\right|$ and $\omega_{s} \neq 0$, (b) for $\left|E_{f}\right| \neq\left|V_{p s}\right|$ and $\omega_{s} \neq 0$ for three different sliding frequencies.

The subtransient armature current can be found by resolving the voltage across the switch GB into two orthogonal components directed along the axes $\mathrm{a}$ and $\mathrm{b}$ as shown in Figure 2. It is important to stress that although the a- and b-axes are directed along the $d$ - and q-axes, the resulting $(a, b)$ voltage and current components have a different meaning to the $(\mathrm{d}, \mathrm{q})$ components. The $(\mathrm{a}, \mathrm{b})$ components are simply the components of the phase quantities resolved along two axes in the complex plane while the $(\mathrm{d}, \mathrm{q})$ voltage and current components are associated with fictitious, rotating, orthogonal armature windings. As the circuit is assumed to be purely reactive, the current must lag the forcing voltage by $\pi / 2$. This means that the a-component voltage $V_{a}$ will force the flow of the b-component of current $I_{b}$ and vice versa [11]. Assuming the switch is switched on at $f_{g}>f_{p s}$, the cumulative kinetic energy of the generator is:

$$
A=\frac{T_{j} \omega_{S}^{2}}{2}
$$

causing the equalizing active power to flow from the generator to the system:

$$
P=\frac{V_{p s}^{2}}{X_{\Sigma}} \sin \delta
$$

As a final result:

$$
\begin{gathered}
\mathrm{A}=\frac{\mathrm{T}_{\mathrm{j}} \omega_{\mathrm{s}}^{2}}{2}=\int_{\delta_{0}}^{\delta_{\max }} \mathrm{P}_{\mathrm{e}} \mathrm{d} \delta=\frac{\mathrm{V}_{\mathrm{ps}}^{2}}{\mathrm{X}_{\Sigma}} \int_{\delta_{0}}^{\delta_{\max }} \sin \delta \mathrm{d} \delta= \\
=\frac{V_{p s}^{2}}{X_{\Sigma}}\left(\cos \delta_{0}-\cos \delta_{\max }\right)
\end{gathered}
$$


where $T_{j}$ is the inertia time constant of the rotating parts of the generator and the turbine, $X_{\Sigma}$ is the total reaction between the two parts: $X_{\Sigma}=x_{d}^{\prime \prime}+X_{T}+X_{p s}, \delta_{0}$ is the starting phase of phase shift between $\overrightarrow{E_{f}}$ and $\overrightarrow{V_{p s}}$ at switch-on time and $\delta_{\text {max }}$ is the maximum offset angle, in which the primary kinetic energy is exhausted and equals the set in the system. In order to maintain stable operation, the angle $\delta_{\max }$ must not exceed the critical value $\delta_{c r}=\pi / 2$. The solution of (7) for $\delta_{\max }$ gives:

$$
\delta_{\max }=\arccos \left(\delta_{0}-\frac{T_{j} X_{\Sigma}}{2 V_{p s}^{2}} \omega_{s}^{2}\right)
$$

The resulting expression indicates that $\delta_{\max }$ is quadratic depending on the slip frequency value and does not depend on the character. If in (8) $\delta_{\max }=\pi \rightarrow \cos \left(\delta_{\max }\right)=$ -1 and $\omega_{\mathrm{s}}=2 \pi f_{c r}$ at known $\delta_{0}$, the critical slip frequency $f_{c r}$ can be determined, whereby the generator can not be held in synchronism.

$$
f_{c r}=\frac{v_{p s}}{\pi} \sqrt{\frac{\left(1+\cos \delta_{0}\right)}{2 T_{j} X_{\Sigma}}}=\frac{v_{p s}}{\pi * \sqrt{T_{j} X_{\Sigma}}} \cos \frac{\delta_{0}}{2}
$$

Equations (8) and (9) are approximations as the asynchronous and reactive moments of the rotor of the generator are not taken into account when their output is checked when the generator is switched on in parallel with the system. Ignoring them does not provide significant reflections for practical purposes.

\section{Alalysis Conclusion}

Taking into account the large differences between the time constants $\mathrm{T}_{J}$ and $\mathrm{T}_{\mathrm{e}}$, the excitation current can be used to influence the rotational speed of the inductor or to vary the slipping between the generator and the grid voltage in the synchronization process. If the turbine spins synchronous aggregate (SA) to revolutions which are same as the required and stops regulating the frequency from the turbine regulator, with small changes in the excitation current, it is simple and fast to change the phase angle between the voltage vectors and thus faster to work synchronously. In order to evaluate the quantitative indicators, the following mathematical study was performed.

\section{MATHEMATICAL ANALYSIS}

In non-loaded mode of the SA, the turbine resistance moment exerts the magnetic field strength only in the air gap between the inductor and the anchor, caused by the excitation current and the mechanical loss of rotation. Impacting the mechanical losses in the synchronization process is virtually impossible. Instead, the excitation current is fully manageable within a relatively wide range. A mathematical model of a hydrogenerator was made to quantify the above-mentioned influence. In the model, the SG is given as an example in [6], as all its constructive parameters are known. The exact values of the factor, the inductions in different parts of the machine, currents, voltages, etc. are available. Thus, the credibility of the mathematical model can be evaluated. The parameters of the modelled synchronous machine are given in Table I and are modelled in FEMM 4.2 [7]. The resistive moment created by the excitation current at the nominal mode and the deviation of $\pm 5 \%$ and $\pm 10 \%$ was calculated. Induction in the air gap [6] is calculated at $0.71 \mathrm{~T}$ at idle and nominal terminal voltages. In the mathematical model, the result is $0.69 \mathrm{~T}$. Figure 4 shows the model of the investigated generator in nominal non-loaded mode and the distribution of the power lines of the magnetic flux and the inductions in the different parts of the machine.

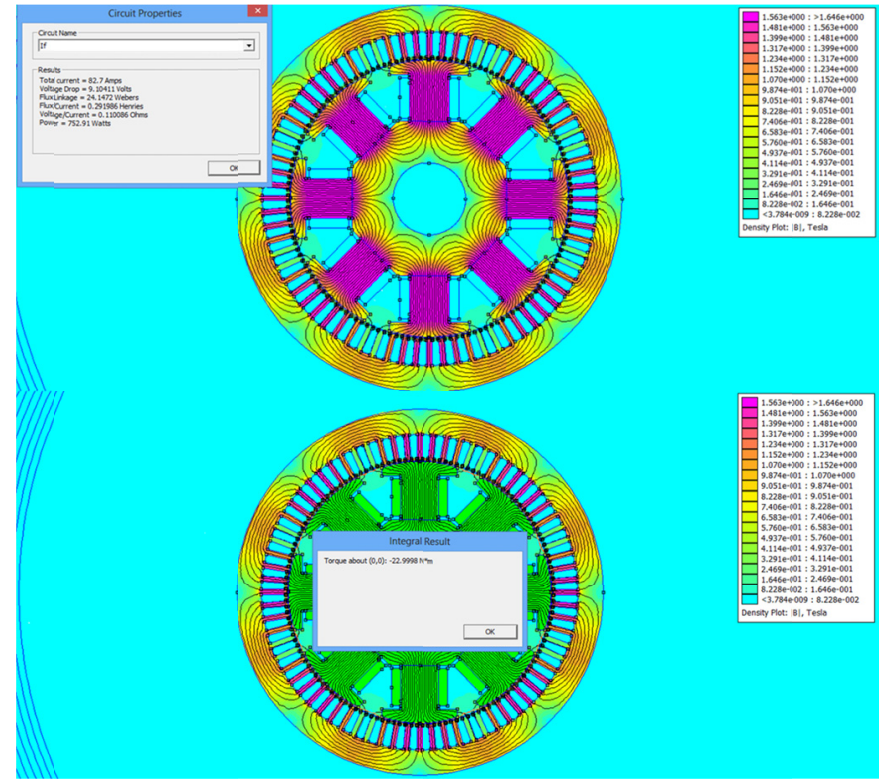

Fig. 4. Model of SG 400kVA / 6,3kV (lower image: resistance torque)

According to the data from hydroelectric power plants to NEK EAD, for hydro sets with Francis turbine and small generators, the opening of the guiding apparatus is about $4 \%$, at 0.9-1 of the nominal speed and non-excited machine. After the excitation is reached and the nominal voltages at the terminals at idling speed of the SG, the opening of the guiding apparatus is approximately $8 \%$. The resistance moment created by the excitation current of the generator is $-23 \mathrm{Nm}$, which is about half of the idling turbine at idle and assumed linear dependence between opening and mechanical power in the range of $4-8 \%$. Table II summarizes the results of the calculations at different excitation current values. The induction distribution in the air gap of the machine is shown in Figure 5. The graph of the resistance of the excitation current is shown in Figure 6. According to the results obtained with the regulation of the excitation current by $5 \%$ of the idle current of the SA, the resistance moment is changed by $1.5 \mathrm{Nm}$, i.e. by $6.6 \%$ of the total. These is a variation of about $24( \pm 12)$ revolutions in unit speed. To achieve accurate synchronization according to [5], slipping $\omega_{s}$ should be no more than 0.01-0.02. In this case, a required synchronous leakage could be equivalent to 7-14 turns per minute. For a change of 14 revolutions, a $0.86 \mathrm{Nm}$ torque, or a change of the rated current from the nominal within $\pm 2 \%$, is necessary. The voltage of the generator terminals will change as well, but slightly. This means that with a change in the voltage, the speed in the synchronization process can be adjusted relatively simple. Due to the physical lack of the above-mentioned generator, in order to validate the resistance 
calculation approach, a mathematical model of a similar synchronous generator in the laboratory of automation of EES in TU-Sofia was created using the same methodology.

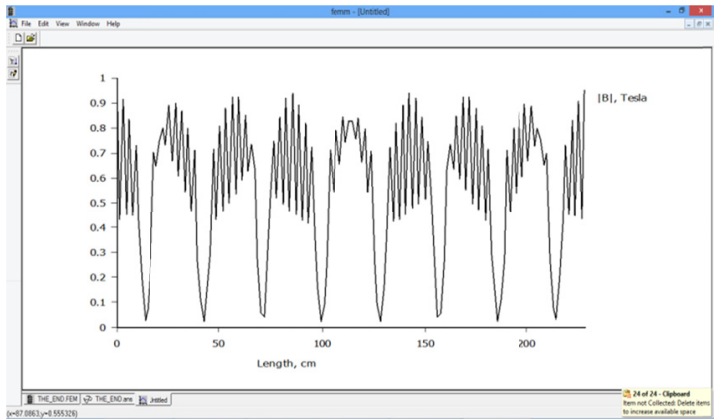

Fig. 5. The induction distribution in the air gap of the machine

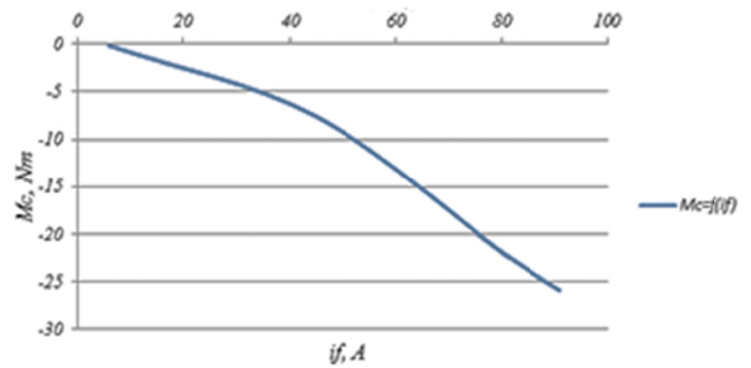

Fig. 6. Changes in the torque resistance of the turbine shaft as a function of the generator output current
TABLE I. PARAMETERS

\begin{tabular}{|c|c|c|}
\hline nominal full power & 400 & $\mathrm{~kW}$ \\
\hline nominal voltage & 6300 & $\mathrm{~V}$ \\
\hline nominal rotational speed & 750 & $\mathrm{~min}^{-1}$ \\
\hline rated power factor & 0,8 & - \\
\hline air gap under the middle of the pole & 4 & $\mathrm{~mm}$ \\
\hline max. air gap under the ends of the pole nozzle & 6 & $\mathrm{~mm}$ \\
\hline axial length & 430 & $\mathrm{~mm}$ \\
\hline outer stator diameter & 990 & $\mathrm{~mm}$ \\
\hline internal stator diameter & 730 & $\mathrm{~mm}$ \\
\hline number of stator channels & 72 & - \\
\hline number of rotor poles & 8. & - \\
\hline number of turns per pole & 41 & - \\
\hline idle current at idling speed & $\mathrm{A}$ & 82,7 \\
\hline excitation current at nominal load & $\mathrm{A}$ & 160 \\
\hline
\end{tabular}

TABLE II. RESULTS

\begin{tabular}{|c|c|c|c|c|c|}
\hline if, $[\mathbf{A}]$ & 75.18 & 78.76 & 82.7 & 86.835 & 90.97 \\
\hline $\mathbf{M c},[\mathbf{N m}]$ & -20 & -21.47 & -23 & -24.50 & -25.90 \\
\hline $\mathbf{B}_{\mathbf{\delta}},[\mathbf{T}]$ & 0.64 & 0.664 & 0.688 & 0.712 & 0.734 \\
\hline $\mathbf{U}_{\mathbf{g}},[\mathbf{k V}]$ & 5724 & 6012 & 6300 & 6390 & 6481 \\
\hline
\end{tabular}

\section{MATHEMATICAL MODELING OF THE SYNCHRONIZATION PROCESS WITH THE EXAMINED GENERATOR}

To complete the above study, a simulation model of a simplified three-phase electric circuit was created (Figure 7) for a part of the small hydropower plant and the connection with the power system with the above-mentioned synchronized generator.

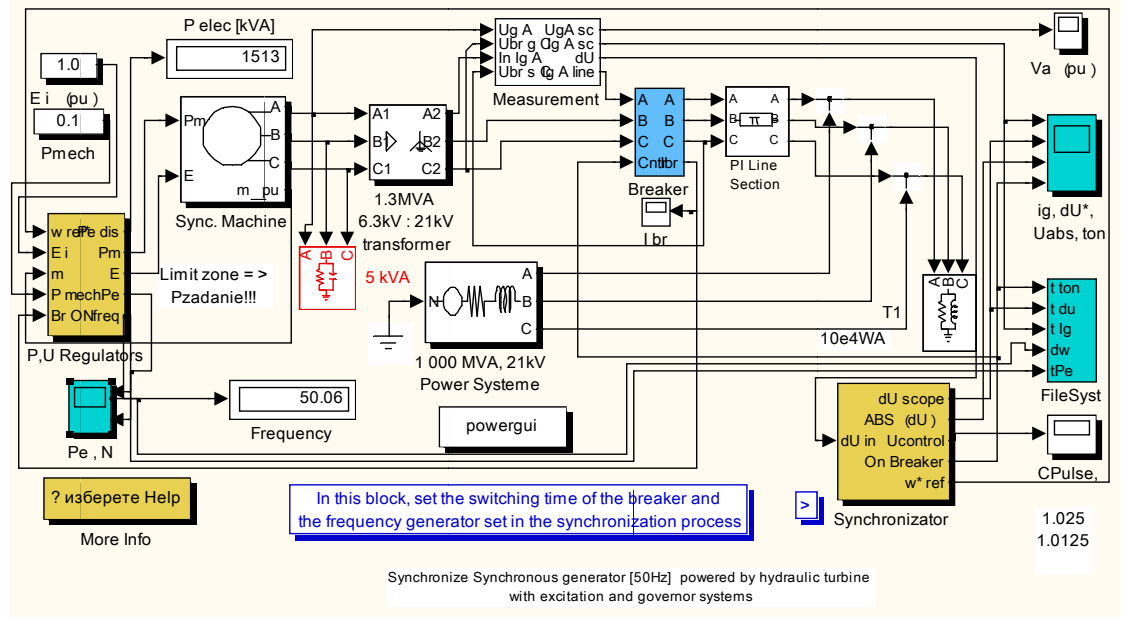

Fig. 7. The Simulink model.

The studies were performed using Matlab in Simulink environment. A Power System Blockset Library was used as well. The generator (Sync_Mashine) has a power of 0.5MVA. The $6.3 \mathrm{kV}$ generator voltage is increased to $21 \mathrm{kV}$ by a Blurry Transformer. The power supply (Power System, "solid-state" voltage) is via a Breaker and a $25 \mathrm{~km}$ (3 Phase Line) power line. Loads are summarized and divided into: internal, for power station (5kVA), and systemic (T1). To facilitate the adoption of the model, the measuring transducers associated with the control are grouped into a separate measurement subsystem. Distinct signals are generated for visualization for all informative values. The current through the breaker and the voltage of the generator are measured with separate oscilloscopes. The speed and voltage regulators of the synchronized generator are grouped together in the "P_U_Regulators" block which is developed specifically for 
the model and performs all regulatory functions. A PID adjustable controller, controls both dimensions. In the setup dialog, the test parameters (generator spinning, excitation moment and secondary equalization) are displayed. There is a built-in control logic in the speed controller, which automatically determines the mode. With the switch off, the control is set to $\mathrm{f}$ (in this case " $\omega$ ", "synchronization" mode). When the circuit breaker is switched on, the regulation is at the active power setting (" $P_{m e h}$ "). In the voltage regulator, the "initial moment of excitation supply" function has been added. This is defined in the dialog mode and set as slippage. The automatic synchronizer is modelled through another specially-developed block, "Synchronizator". The logic functions set forth fully correspond to the requirements for SG synchronization devices with a combined method with control of optimal sliding period - checking the conditions, controlling the parametersand triggering the impulse to switch on the circuit breaker. Their settings are set in dialog mode. The control of the current offset angle value is based on the measurement of $d U$ and the instantaneous angular velocity. The frequency of the power system is $50 \mathrm{~Hz}$. In addition, there are modelled functions for setting the allowed angular speed for synchronization $\omega_{\text {optimal }}$ and switching (trip) time $t_{\text {onbreaker }}$. The block has an input signal proportional to the beating voltage. Outputs of the block are the signals for the circuit breaker control, reference for the speed governor and the voltage display $d U$ and circuit breaker status. In the initial state of the model, the circuit breaker is off and the synchronized generator is disconnected. The initial angular velocity is determined by the "Sync_Mashine" setting. If a synchronization process with the power system is started, the duration depends on the selected starting and permissible speed, as well as the settings of the turbine PID regulator. After switching on the circuit breaker, the power delivered by the synchronized generator is regulated. The feature of the regulator is static $(1 \%)$.

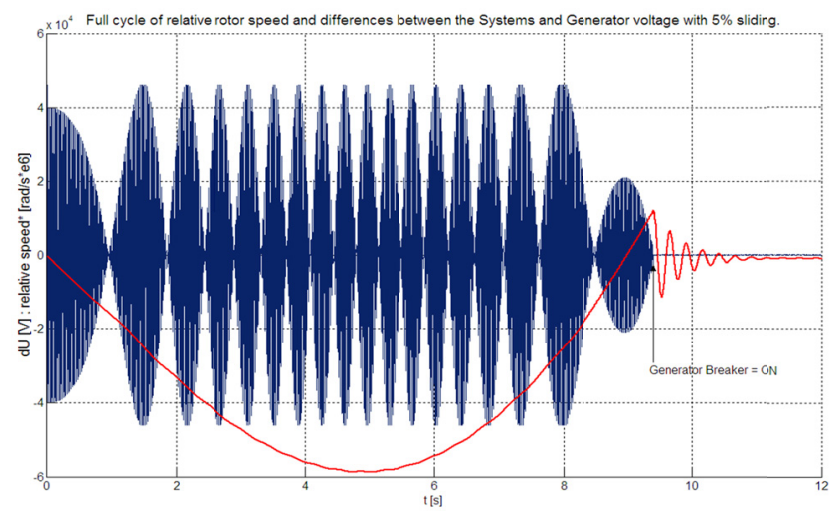

Fig. 8. Full cycle of relative rotor speed and differences between the system and generator voltage with $5 \%$ sliding

Through the examined model, the possibility of fine-tuning the speed of the synchronization process through the excitation regulator is investigated. Its effect is the rotor rotation compensation of the synchronized aggregate by an additional in-phase amplitude modulation of the excitation current's current value. The structure of the "P U Regulators" is changed so that after the generator has rotated to a nominal speed and an excitation has been given when the two zones are reached:

- for the speed control regulator: the generator's rotation speed causes the specified slips for synchronization

- for the excitation regulator: voltage that gives us a difference of $U_{g}$ and $U_{\text {sys }}$ within the permissible,

The algorithm of action is changed as follows:

- The "variable speed" speed controller switches to "job setting" and maintains constant output. The mechanical power from the turbine to the generator is established and becomes constant.

- Switch the excitation control from the voltage maintenance mode to the generator voltage control mode. The goal is to maintain a constant slip between the voltage vector of the system and the generator $\left(\omega_{\mathrm{s} 0}=\right.$ const $)$ with the faster regulator. This would satisfy the basic requirement even for synchronizers operating with the constant angle of the overtaking method, with a permissible difference in amplitudes of stress vectors.

Studies are conducted at a constant value of the mechanical power output and different values of the excitation current (for the Matlab model: excitation voltage). The range of excitation current $\left(I_{\text {fnon-loaded }} \equiv I_{f n l}\right)$ variation is consistent with the results of previous mathematical modelling: 0.925I $I_{f n l}-$ $1.1 I_{f n l} . I_{f n l}$ is the excitation current of the idle generator at which $U_{g}=U_{s y s}$ and $\omega_{s}=\omega_{0}$. The step between succesive values is $0.025 I_{f n l}$. We set the maximum idle power of the generator to mechanical power $P_{\text {mech }}=0.1 * P_{\text {mech_nom }}=$ const. Informative parameters in the study will be the impact of the applied principle on:

a) Main Indicators: The relative change of the dwelling period $T_{s}=\frac{1}{f_{s}}=\frac{2 \pi}{\omega_{s}}$ of $d U$ and the depth of rotor fluctuations and time of damping.

b) Restrictive Conditions: Maximum generator voltage and voltage difference between the system and the generator at the moment of switching on and the ustainable parallel entry, i.e. magnitude of the equalizing current (electrical power).

The study results are shown in Figures 9-12. The base of [pu] time " $t_{-} d U$ " is for " $\left|E_{f}\right|=\left|V_{p s}\right|$ ". The speed controller works in mode "regulation by setting" and maintains $10 \%$ of the nominal mechanical power. We notice a general change in the period of the low-frequency component of voltage dU as shown in Figure 9. Figure 10 demonstrates the change of period and amplitude of beating voltage $\mathrm{dU}$ for the limit and nominal values of $I_{f n l}\left(0.925 I_{f n l} ; 1.0 I_{f n l} ; 1.1 I_{f n l}\right)$. The deviation of the rotational speed of the rotor [pu] in the process of timing synchronization with excitation for the limit and nominal values of $I_{f n l}$ and the electrical power $\mathrm{P}_{\mathrm{e}}[\mathrm{pu}]$ in the synchronization process with excitation control for the limit and nominal values of $I_{f n l}$ are shown in Figures 11 and 12 respectively. 


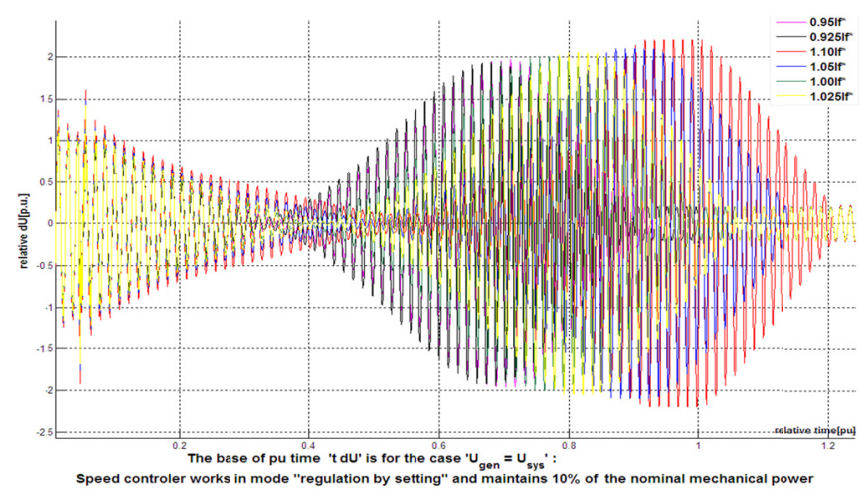

Fig. 9. Dependence of sliding period $T_{s}$, $[\mathrm{pu}]$ by excitation current $I_{f n l}$

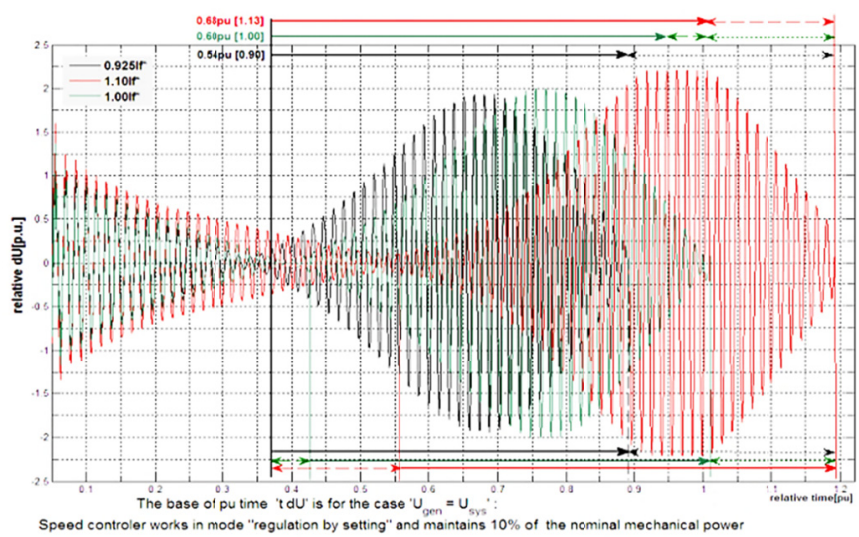

Fig. 10. Dependence of sliding period $\mathrm{T}_{\mathrm{s}},[\mathrm{pu}]$ by nominal and limits values of excitation current $I_{f n l}$

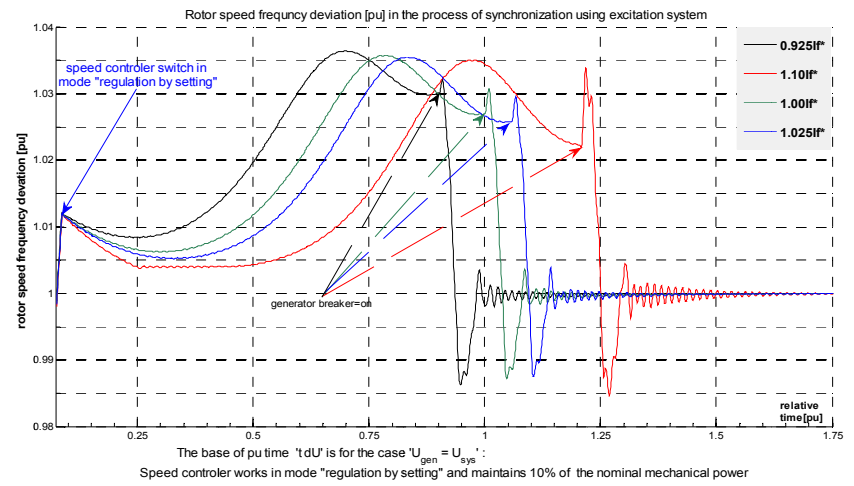

Fig. 11. Rotor speed frequncy deviation [pu] before and after breaker closing in the process of synchronization using excitation system

The result analysis shows that the effect of the applied management principle is tangible with regard to all key indicators and restrictive conditions. The best results are achieved at the over-synchronous rotation speed at $1.025 I_{\text {fnl }}$ and $1.05 I_{f n l}$. The process at $1.025 I_{f n l}$ is optimal, which is practically equivalent to that of $1.0 I_{f n l}$ in terms of dynamics. Its essential advantage is that the excitation regulator has practically performed a "forcing" of the excitation with $20 \%$
$I_{f n l}$ after switching on the generator breaker - an action that creates a damping torque and ensures that the critical value of the power angle $\theta_{c r}$ will not be surpassed.

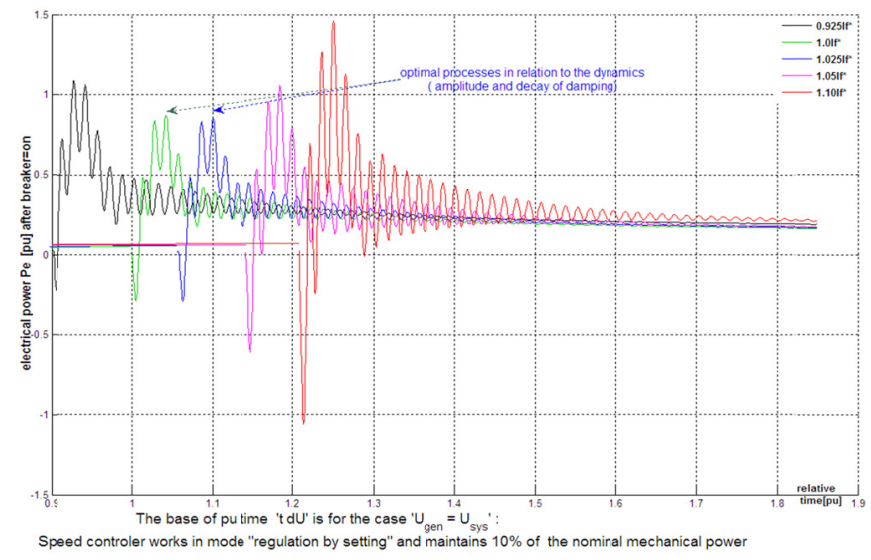

Fig. 12. The electrical power $P_{e}[\mathrm{pu}]$ in the synchronization process with excitation control for the limit and nominal values of $I_{f n l}$

\section{SLIP CONTROL WITH A DIGITAL SYNCHRONIZER}

In this case, the maintenance of the optimal sliding of the voltage vectors is controlled by the synchronizer. The standard digital outputs of the synchronizer are connected to the inputs of the speed controller. Excitation adjustment is achieved by connecting the outputs of the digital synchronizer not to the inputs of the speed controller but to the digital inputs of the excitation system. The signal for increasing the rotation speed is entered to the excitation reduction input, and for speed reduction to the excitation input. The following additional restrictive conditions shall be taken into account:

- The speed control signal remains at the last level after reaching the optimum synchronization zone, i.e. the speed controller is already in "on-demand" mode.

- Changing the excitation in this situation means that the circuit breaker will close its contacts at voltages that are generally of varying amplitude. If this difference is within the permissible tolerance of $2.5 \%$, it can be assumed that the equalizing current due to the voltage difference is just one part of the initial jet load after the parallel input. This load is a consequence of the slight forcing of the excitation to keep the generator in synchronicity.

- It is likely to reach the maximum generator voltage limit with this principle of regulation. It is then necessary to position the output of the speed regulator to a lower level and continue the algorithm in the same way.

- If a minimum excitation limitation occurs, the speed controller is set one level up.

In order to avoid the situations of the last two constraints, and to make this algorithm as realistical as possible, it is necessary to advance the following requirements for the operation of the SA regulators after the activation of the synchronization device: 
- For the excitation regulator, the reference is no longer equalizing the voltages on both sides of the circuit breaker, but it is working on a changing assignment of magnificating or reducing inputs.

- Protections for maximum generator voltage and minimum excitation are mandatory, as a signal to the control system is provided when reaching any of the limitations.

- The synchronization action enables signal switches that put the speed controller to the "on-demand" mode.

- The synchronization action must be resolved after an analysis of the possible adjustable voltage range, i.e. the optimal option is that the speed controller is "locked" at a generator voltage close to the rated voltage.

\section{SLIP CONTROL WITHOUT A DIGITAL SYNCHRONIZER}

In this case, optimal frequency slipping is controlled by the excitation regulator. It is necessary to implement the following requirements for the operation of the SA regulators after the activation of the synchronization device (or algorithm):

- For the excitation regulator the reference is no longer equalizing the voltages on both sides of the circuit breaker, but the frequency of the generator voltage corresponding to the set sliding.

- The functions of the synchronizer are depleted by selecting the optimum switching torque (regardless of the method).

- The other three requirements remain valid.

The developed software for the defined algorithm is programmed into the laboratory physical bench, which can actually test the processes.

\section{RESEARCH RESULTS}

After the adjustments to the algorithms of the regulators with the physical stand, studies were conducted. Changes have been made so that the excitation controller controls the slip (Option 2). The digital synchronizer has a precise synchronization algorithm with a constant time of overtaking. The processing of the entries from the built-in registrar was done with the REGFX32 software [4, 12]. The particular physical model has a clearly expressed slot harmonic. Nevertheless, this does not affect the quantitative and qualitative assessment of the results.

\section{1) Effect of the excitation current on the idle generator frequency.}

The excitation controller is astatic. An idling attempt is made at the acoustic setting of the speed controller and the jump of the excitation controller. Figure 13 shows the strong influence of the excitation current on the speed.

\section{2) Influence of rotor speed on the idle generator voltage.}

The speed controller is astatic. An idling attempt is made at an acoustic setting of the excitation controller and a jumpchange of the rotor speed set point. Figure 14 shows the PWM control by controller, the changes in the excitation current of the SG and as a result the change in the frequency of the generator voltage. A $10 \%$ change of excitation current leads to a $0.17 \mathrm{~Hz}$ change of SM tension frequency. The results show that the inconsistent action of the individual regulators in both cases lead to a hesitation in the process.

\section{3) Synchronization process with coordinated action of regulators.}

Once the adjustable range has been reached, the speed governor is in "idle" mode, maintaining constant torque through the electromechanical drive. The excitation regulator provides the set slip between the voltage vectors of the SG and power system. The digital synchronizer includes a breaker (at 213920ms).

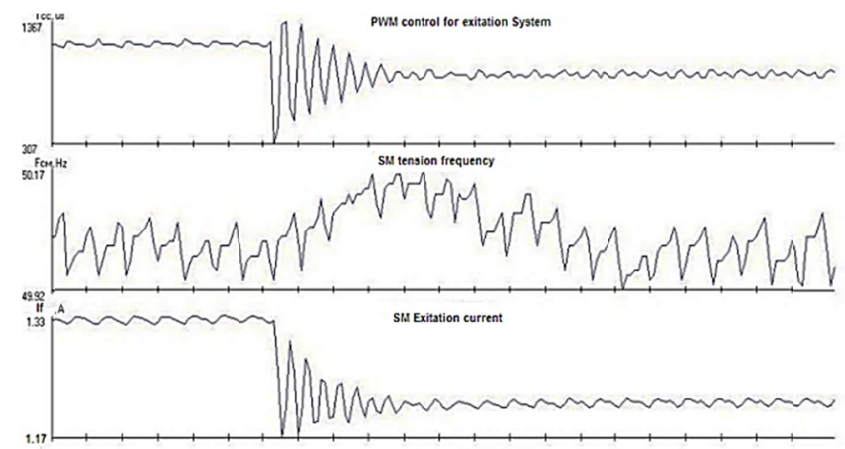

Fig. 13. Impact of the excitation current on the idle generator frequency

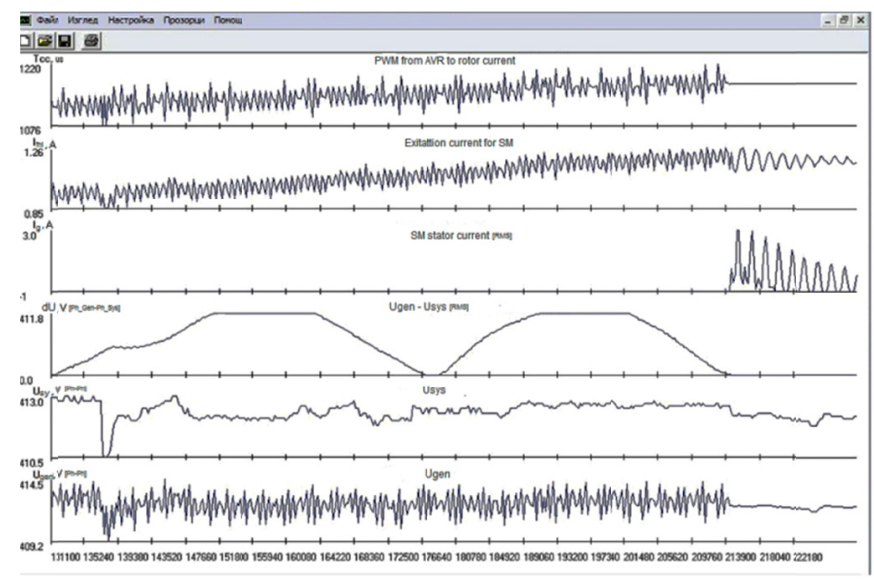

Fig. 14. Entering parallel of SG with power system - two cicles of dU[RMS] by excitation system sliding control.

\section{RESULTS ANALYSIS AND CONCLUSIONS}

With synchronization using the excitation current to finetune the revolutions, an optimal parallel entry time can be achieved with minimal exchange kinetic energy, i.e. fulfilling the basic requirements for minimum equalizing current and oscillating the angle between the stator field and the position of the rotor within the permissible range. For this purpose, changes were made to the structure and configurations of the 
excitation regulators and the rotational speed of the SA. An allin-one method is used- three parallel algorithms for speed excitation and synchronization control in one microcontroller. In the physical model in the laboratory, the algorithm works correctly. The results of the synchronization process are satisfactory, which justifies the algorithm being tested on a real physical object, provided that it has full access to and control over the excitation system algorithms, the speed control system, the digital synchronizer, and the general-station control. The all-in-one principle is optimal for small HPP, given that algorithms work with the same input parameters, with only output control signals directed to different objects with different time constants. The change of the algorithms for the control of different processes in real time is done simply and reliably. This topic could be examined in future research.

\section{REFERENCES}

[1] И. Н. Попов, Обща елетротехника, част 3 , Синхронни и асинхронни машини, С., Наука и Изкуство, 1951

[2] R. Richter, Elektrische Maschinen II, Springer Basel AG, 1953

[3] J. Machowski, J. Bumby, Power System Dynamics, Stability and Control, John Wiley \& Sons Ltd, 2008

[4] A. Tsolov, Power System automation - processes analisis and system automation, Avangard Prima, 2014

[5] Н. И. Овчаренко, Автоматика энергосистем, Учебник для вузов, 3-е изд, Издательский дом МЭИ, 2009

[6] J. Chiasson, Modeling And High Performance Control Of Electric Machines, John Wiley \& Sons, 2005

[7] D. Meeker, Finite Element Method Magnetics, Version 4.2, User's Manual, 2013

[8] D. Dobrilov, A. Tsolov, "Study on the excitation current influence on idle running Synchronous Generator shaft braking torque - mathematical model", CEMBEF 2015, Nis, Serbia

[9] IEEE Std 1010-1987, IEEE Guide for Control of Hydroelectric Power Plants, in: IEEE Std 1010-2006 (Revision of IEEE Std 1010-1987), 2006

[10] IEEE Standard C 50. 12-2005 for Salient-Pole $50 \mathrm{~Hz}$ and $60 \mathrm{~Hz}$ Synchronous Generators and Generator/Motors for Hydraulic Turbine Applications Rated 5 MVA and Above, 2005

[11] IEEE Std C37.118.1, IEEE Standard for Synchrophasor Measurements for Power Systems, 2011

[12] A. Tsolov, "Stand-Simulator for the study of processes and digital regulators", Proceedings of the Technical University - Sofia, Vol. 60, Book 1, pp. 172-178, 2010

[13] D. Dobrilov, A. Tsolov, "Study of factors affecting tension in the point of connection of small scale hidro", EF Conference, TU-Sofia, Book 1, pp. 21-26, 2012 